\title{
MHD SIMULATIONS OF JETS FROM ACCRETION DISKS: NONSTEADY JETS VS. STEADY JETS
}

\author{
T. KUDOH AND K. SHIBATA
}

National Astronomical Observatory, Mitaka, Tokyo 181, Japan $<k u d o @ s o l a r . m t k . n a o . a c . j p>,<$ shibata@solar.mtk.nao.ac.jp >

AND

\section{R. MATSUMOTO}

Department of Physics, Faculty of Science, Chiba University Inageku,Chiba263, Japan <matumoto@c.chiba-u.ac.jp>

We present the results of 2.5-dimensional MHD simulations for jet formation from accretion disks in a situation such that not only ejection but also accretion of disk plasma are also included self-consistently. Although the jets in nonsteady MHD simulations (e.g., Uchida \& Shibata 1985, Shibata \& Uchida 1986, Matsumoto et al. 1996) have often been referred to as transient phenomena resulting from a special choice of initial conditions, we found that the characteristics of the nonsteady jets are very similar to those of steady jets: (1) The ejection point of the jet, which corresponds to slow magnetosonic point in steady MHD jet theory, is determined by the effective potential which results from the gravitational force and the centrifugal force along a field line (Blandford \& Payne 1982). (2) The dependence of the velocity $\left(v_{z}\right)$ and mass outflow rate $\left(\dot{M}_{w}\right)$ on the initial magnetic field strength is about $\dot{M}_{w} \propto B_{0}$ and $v_{z} \propto\left(\Omega_{F}^{2} B_{0}^{2} / \dot{M}_{w}\right)^{1 / 3}$, where $B_{0}$ is an initial poloidal magnetic field strength, and $\Omega_{F}$ is an 'angular velocity of the field line' which is nearly the same as the Keplerian angular velocity where the jet is ejected. These are consistent with those of 1D steady solution (Kudoh \& Shibata 1997), although the explanation is a little complicated in the $2.5 \mathrm{D}$ case because of an avalanche-like accretion. We also confirm that the velocity of the jet is of order of the Keplerian velocity of the disk for a wide range of parameters. We conclude that the ejection mechanism of nonsteady jets found in the 2.5-dimensional simulations are understood with a previous theory which is studied on the assumption of steady state 


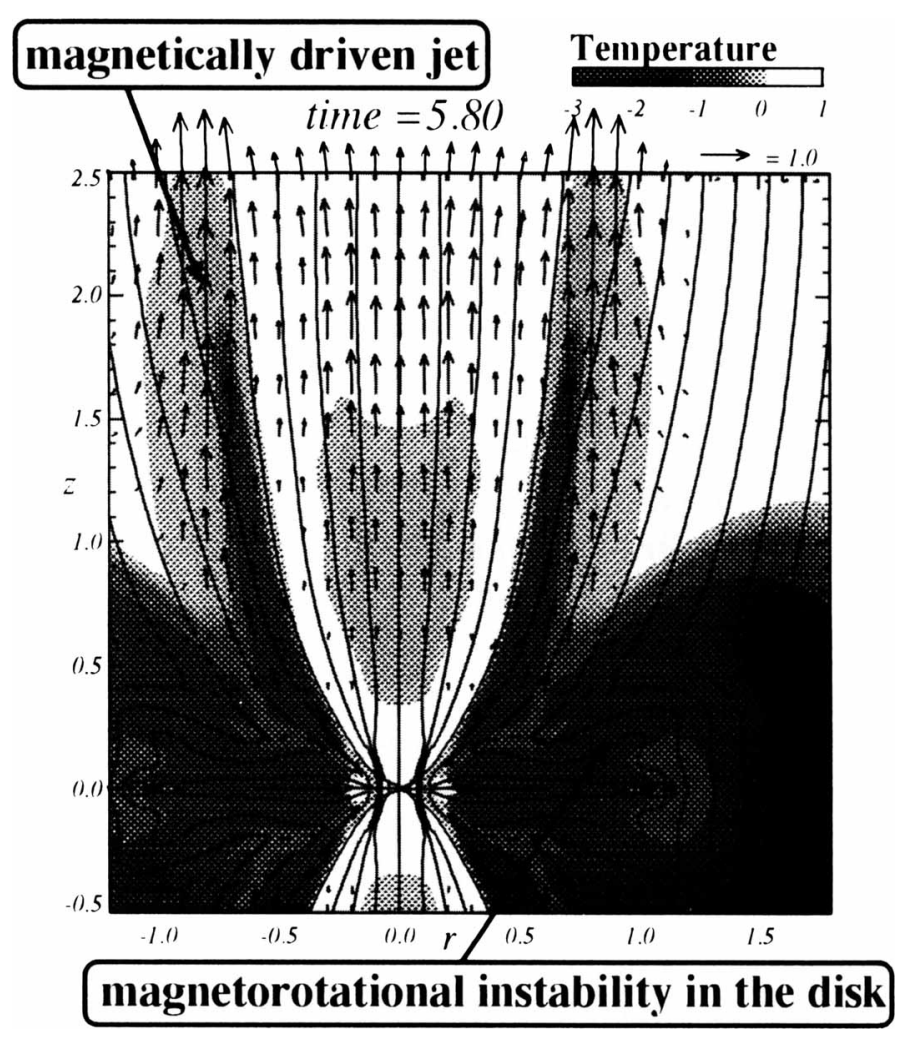

Figure 1. Temperature distribution at $t=5.8$. Time $t=2 \pi \simeq 6.28$ corresponds to one Keplerian orbit at $(r, z)=(1,0)$. Arrows show the poloidal velocity vectors normalized by the Keplerian velocity at $(r, z)=(1,0)$. Solid lines are magnetic field lines. The parameter is $E_{\mathrm{mg}}=2 \times 10^{-3}$, where $E_{\mathrm{mg}}=\left(V_{\mathrm{A} 0} / V_{\mathrm{K} 0}\right)^{2}, V_{\mathrm{A} 0}$ is the Alfvén velocity, and $V_{\mathrm{K} 0}$ is the Keplerian velocity at $(r, z)=(1,0)$. Magnetically driven jets are ejected from a thick disk. The magnetorotational instability (Balbus \& Hawley 1991) is occurring in the disk.

even when nonsteady avalanche-like accretions occur along the surface of disks.

\section{References}

Balbus, S.A., \& Hawley, J.F. 1991, ApJ, 376, 214

Blandford, R. D. \& Payne, D. G. 1982, MNRAS, 199, 883

Kudoh, T., \& Shibata, K. 1997, ApJ, 474, 362

Shibata, K., \& Uchida, Y. 1990, PASJ, 42, 39

Matsumoto, R., Uchida, Y., Hirose, S., Shibata, K., Hayashi, M. R., Ferrari, A., Bodo, G., \& Norman, C. 1996, ApJ, 461, 115

Uchida, Y., \& Shibata, K. 1985, PASJ, 37, 515 